\title{
Surface Deformation Monitoring of a Section of Gongyu Expressway Based on SBAS-InSAR Technology
}

\author{
Ying Yang ${ }^{1,2}$, Yifang Sun ${ }^{1,3}$, Shihong $\mathrm{Wu}^{2}$, Xuegang Dong ${ }^{1}$, Hanyao Huang ${ }^{1}$, Xiaoxu Cao ${ }^{1}$, Xinqing Huang ${ }^{1}$, and Zhiguo \\ Meng ${ }^{1,3 *}$ \\ ${ }^{1}$ College of Geoexploration Science and Technology, Jilin University, Changchun, Jilin, 130026, China \\ ${ }^{2}$ Tianjin Research Institute for Water Transport Engineering M.O.T., Tianjin, 300456, China \\ ${ }^{3}$ Institute of National Development and Security Studies, Jilin University, Changchun, Jilin, 130026, China
}

\begin{abstract}
It is difficult to monitor the surface deformation along the expressway for the critical climate conditions in Tibet plateau. In this paper, based on sentinel-1A SAR data, the surface deformation along the Gongyu expressway was tried to evaluate using time-series SBAS-InSAR method. The results indicate that the surface deformation in most regions is within the safe acquirement of the expressway. Moreover, the surface deformation indicates a strong seasonal effect. Finally, two special spots with dangerous surface deformation are identified along the expressway.
\end{abstract}

\section{Introduction}

Gonghe-Yushu Expressway, referred to as Gongyu Expressway, has a total length of 634.8 kilometers, of which permafrost is 227 kilometers long. It is the first expressway in China that crosses the Qinghai-Tibet Plateau and also the first expressway constructed in the permafrost region. Thus, it has great referring significance for China's future transportation engineering construction in high altitude and alpine regions [1-3].

Traditional surface deformation monitoring methods, including levelling, ground survey, GPS observation, are easily affected by the weather [4]. Moreover, the real-time data tends to be insufficient due to the high work intensity. The elevation accuracy of GNSS observation technology is low, and some satellite signals may become unmeasurable due to obstruction of ground objects. Therefore, none of the above methods can simultaneously guarantee all-weather, high-precision, large-area deformation monitoring of ground objects.

Differential Interferometric Synthetic Aperture Radar (D-InSAR), as a new type of surface deformation monitoring method, has the above three advantages [5-6]. Therefore, D-InSAR technology has been widely used to monitor the surface deformation. Zeng et al. verified the applicability of Sentinel-1A data in the study of frozen soil in high latitude regions [7]. $\mathrm{Xu}$ summarized the characteristics of the InSAR method in monitoring the settlement of Shenzhen urban roads and subway [8]. Li et al. evaluated the deformation of a mountain and the surface of an expressway, verifying the effectiveness of the InSAR technology in monitoring the deformation along the high-speed railway [9]. Luo et al. used the InSAR technology to study the physical properties and the deformation mechanism of permafrost in the Qinghai-
Tibet Plateau [10]. However, this technology was seldom used to monitor the deformation of the linear objects such as highways in alpine permafrost regions such as the Tibet Plateau, where the complex climate makes research more complicated and difficult.

This paper selects a section of the Gongyu Expressway, which starts from Chapcha Town, Gonghe County, and ends at the point that is about 2.5 kilometers away from Qinggenhe Village, Xinghai County, and uses 48 Sentinel-1A images from March 2017 to May 2020. For the first time, the SBAS-InSAR technology was used to monitor the surface deformation of the area, and the cumulative deformation and deformation rate was obtained, and the influence of frozen soil and lithology on the surface deformation of the area was discussed.

\section{Study area and data source}

\subsection{Study area}

In this study, the selected section of the Gongyu Expressway starts from Chapcha Town, Gonghe County, and ends at the point, which is about 2.5 kilometers to the southeast of Qinggenhe Village, Xinghai County (Figure $1)$. The section is about 130 kilometers long, ranging from $99^{\circ} 33^{\prime} \mathrm{E}$ to $100^{\circ} 33^{\prime} \mathrm{E}$ and from $36^{\circ} 19^{\prime} \mathrm{N}$ to $35^{\circ} 46^{\prime} \mathrm{N}$. The northeastern part of the study area is relatively flat, mostly cultivated land and bare land. The southwest part is the Kunlun Mountains. 


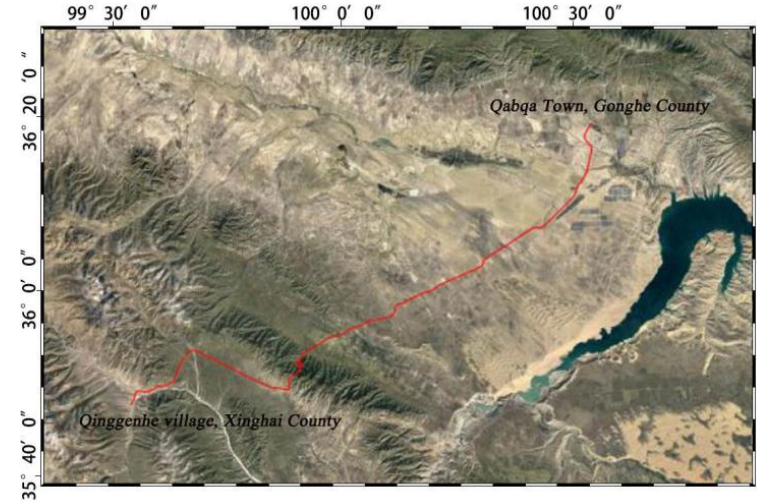

Figure 1. Location of the study area.

This Expression section belongs to the seasonally frozen soil area of the Tibet Plateau, which is sensitive to the climate changes. The seasonal frost heave and thaw collapse of the frozen soil layer, resulting in frost heave mounds and other phenomena. Therefore, studying the surface deformation of this area also has a good reference value for exploring the influence of seasonal changes in frozen soil on the deformation of artificial linear features.

\subsection{Data source}

In this paper, 48 scenes of C-band SAR data on the same descending orbit acquired by the Sentinel-1A satellite between 2017 and 2020 were employed. The incident angle is $42.1^{\circ}$, and the spatial resolution of the data is 5 $\mathrm{m} \times 20 \mathrm{~m}$. The observation mode is interference width (IW), and the polarization mode is single polarization (VV). The time span of the used data is 1140 days, and the interval of each scene is about 24 days.

The SRTM 1 DEM data with a spatial resolution of 30 $m$ downloaded from the NASA official website was used as the reference for SAR image processing. The positioning accuracy is $20 \mathrm{~m}$, the relative elevation accuracy is $10 \mathrm{~m}$, and the absolute elevation accuracy is $16 \mathrm{~m}$. In order to ensure the orbital accuracy of the Sentinel data and to eliminate the flawless baselines and residual fringe errors caused by orbital errors, the Precise Orbit Ephemecrides (POD) data published by ESA was used in this experiment, whose positioning accuracy is better than $5 \mathrm{~cm}$.

\section{Data processing}

Small Baseline Subset (SBAS) is a new time series analysis method proposed by Berardino and Lanari for studying low-resolution, large-scale deformation [11]. The basic principle is to use the deformation result obtained by a single D-InSAR as the observation value, and then to estimate a high-precision deformation time series based on the least squares rule. In this paper, ENVI SARscape software was used to operate the SBAS-InSAR method. Interference, leveling, filtering, phase unwinding, orbit refining, inversion and geocoding were carried out for 48 sentinels-1A images covering the study area from March 2017 to May 2020. The specific processes to acquire temporal surface deformation rate in the study area can be summarized as follows:

(1) Connection graph generation. The SAR image acquired on June 29, 2017 was used as the super master image for registration. After several experiments, the spatial baseline threshold of the interference pair connection was set to $2 \%$ of the critical baseline, and the time baseline threshold is 105 days. A total of 176 interference pairs are generated.

(2) Interfere workflow. The coherence of 176 paired images was calculated, and the first flattening was conducted in this step. After several experiments, the commonly used Goldstein method was adopted to filter the images to reduce noise. The threshold value of the unwinding correlation coefficient was set as 0.15 , and the phase unwinding was carried out using the classical minimum cost flow (MCF) method. After checking the coherence coefficient graphs and phase unwrapping graphs of all the image pairs, the image pairs with large area of low coherence or unsatisfactory unwrapping results were removed and filtered. Finally, 144 interference pairs were retained.

(3) Rail refining and re-leveling. Via Google Earth, 597 relatively stable control points were selected from buildings and exposed rocks in the study area. After multiple errors calculating and screening, 124 ground control points were identified, and the absolute residual values were kept less than 1.0, which ensured the accuracy of control point selection.

(4) SBAS analysing. According to the common coherence values, the correlation coefficient threshold is adjusted to 0.25 , and the MCF method is used for secondary unwrapping to optimize the interference results. Checking all the unwrapping results, the image pairs with unsatisfactory understanding are eliminated and 99 pairs of interference pairs are retained for subsequent calculation. Thereafter, the high-pass and low-pass filters are used to remove atmospheric phase. The size of spatial correlation distribution filter window is 1200 meters, and the size of temporal correlation distribution filter window is 365 days. 124 GCPs are used to remove residual phase or phase slope in orbit refining.

(5) Geocoding. The deformation rate and cumulative deformation variable are transformed from SAR coordinate system to geographic coordinate system. The threshold value of height accuracy is $9 \mathrm{~mm}$ and that of deformation rate is $4.5 \mathrm{~mm} / \mathrm{a}$. The results are projected along the vertical direction.

\section{Results and discussions}

Taking 0.25 as the coherence threshold, the average annual deformation in the LOS direction is obtained. The statistical results show that the values of the average deformation rate are mainly from $-4.5 \mathrm{~mm} / \mathrm{a}$ to $4.5 \mathrm{~mm} / \mathrm{a}$. Therefore, to improve the accuracy and reliability of the results, the control elevation accuracy threshold is set as $9 \mathrm{~mm}$ and the deformation rate accuracy threshold is set as $4.5 \mathrm{~mm} / \mathrm{a}$ in geocoding step. Then, the output is only the points whose accuracy met the threshold. At last, the 
average annual deformation rates in LOS and vertical directions are generated (Figure 2).

Since the accuracy threshold is set as $4.5 \mathrm{~mm} / \mathrm{a}$, the position of $\pm 4.5 \mathrm{~mm} / \mathrm{a}$ is regarded as basically stable and not as surface deformation. Figure 2 shows that the surface deformation in most areas along the Gongyu Expressway are stable. But the following three phenomena should be mentioned.

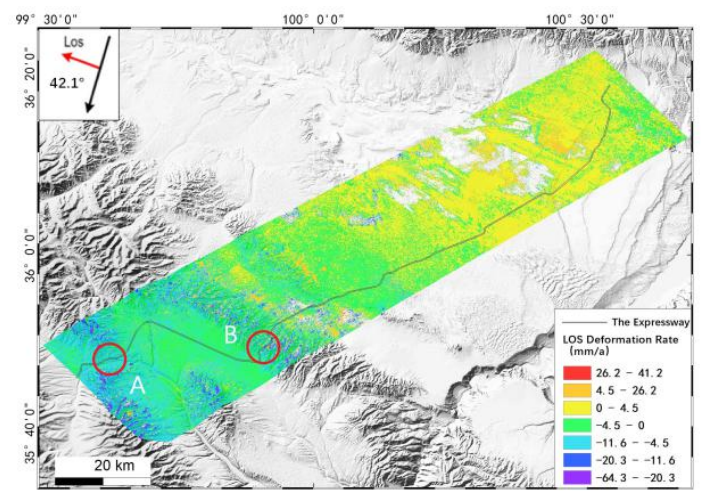

First, the apparent surface depression occurs in the southern part of the expressway, particularly the high mountains not far away from the road.

Second, the clear surface uplift exists in the northern part of the expressway, particularly in the regions centred at $\left(100^{\circ} 25^{\prime} \mathrm{E}, 36^{\circ} 10^{\prime} \mathrm{N}\right)$. Also, the uplift is not far away from the road in the north part.

Third, the mountains indicate a complex surface deformation.

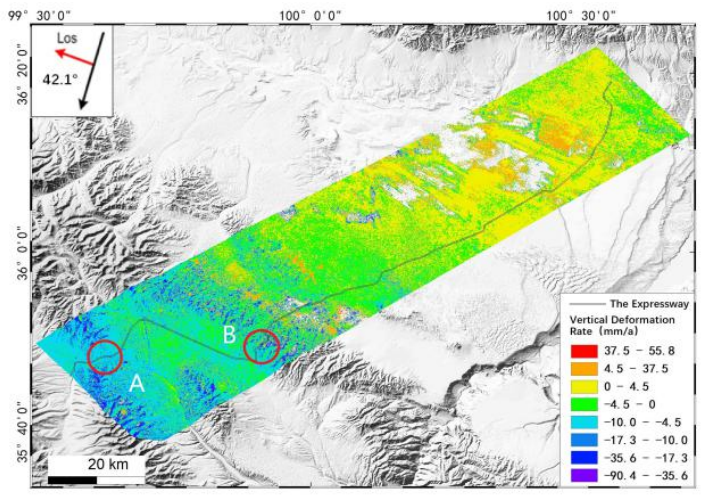

Figure 2. Average annual deformation rate in the LOS direction (a) and vertical direction (b).

Moreover, the mountain area is potentially the most dangerous region for the safe operation of the expressway. Thus, to better understand the surface features along the

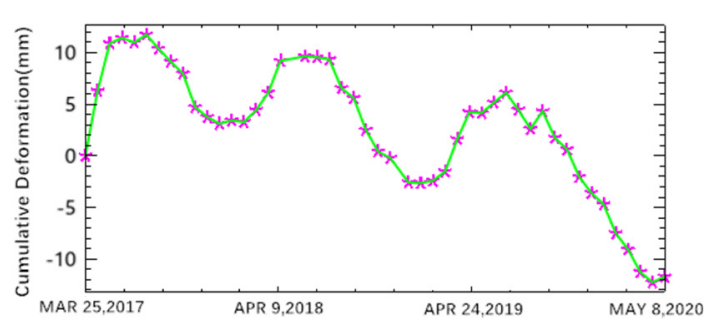

expressway, the time series deformation curves of two special spots in the mountains, marked A and B in Figure 2, are generated (Figure 3 ).

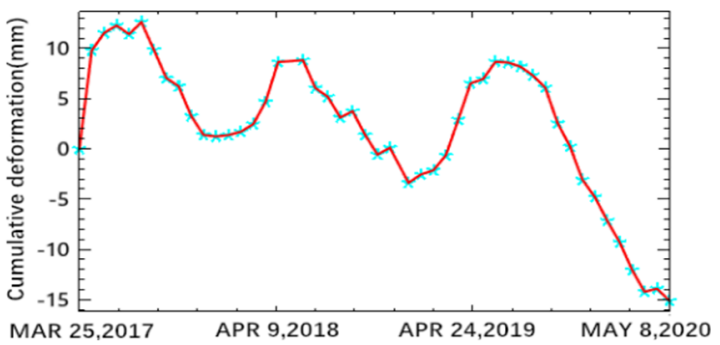

Figure 3. Time series deformation curves of Spots A (left) and B(right).

Figure 3 shows a similar time series deformation. From March to June to 2017, the surface deformation was apparently increased. After that, the surface deformation was monotonously depressed until January of 2018. Then, the surface deformation was monotonously increased until May of 2018. After that, the surface deformation was monotonously declined until December, 2018. Thereafter, the surface deformation was again monotonously increased until May and June of 2019. However, the surface deformation was monotonously declined until May of 2020. Thus, two conclusions can be summarized. First, the change of the surface deformation is seasonal. Second, the continuous depression from 2019 to 2020 hints the destroy of the road, which is dangerous for the safe operation of the expressway.

\section{Conclusions}

In this paper, based on Sentinel-1A/B data from 2017 to 2020, the surface deformation of the expressway from Gonghe to Yushu was firstly evaluated using time-series SBAS-InSAR method. The results indicate that the surface deformation in most region of the study area is stable, and it is not dangerous for the safe operation of the railway. Moreover, the surface deformation indicates a strong seasonal effect, which is largely increased from December to June of the next year and depressed from Jun to December. However, two special spots in the mountain area are identified, which are dangerous for the safe operation of the Gongyu expressway.

For the limitation of the spatial resolution of the used Sentinel data, the surface deformation in the expressway or the nearby regions is difficult to identify. More work should be done to keep the safe operation of the railway.

\section{Acknowledgments}

The Sentinel-1 A data was downloaded from https://search.Earth-data.nasa.gov/search. This work was supported by the Science and Technology Project of Qinghai Provincial Transportation Department (Grants No. 2019-5). 


\section{References}

1. Bardou, E., Delaloye, R. (2004) Effects of ground freezing and snow avalanche deposits on debris flows in alpine environments. Nat Hazards Earth Syst Sci., 4(4): 519-530.

2. Zhang, W. (2013) The general situation of Tibet natural disaster and the development process of its disaster aid. J. Catastrophology, 28(4): 176-181.

3. Delaney, K.B., Evans, S.G. (2015) The 2000 Yigong landslide (Tibetan Plateau), rockslidedammed lake and outburst flood: review, remote sensing analysis, and process modelling. Geomorphology, 246: $377-$ 393.

4. Ouyang, C.J., An, H.C., Zhou, S., Wang, Z.W., Su, P.C., Wang, D.P., Cheng, D.X., She, J.X. (2019) Insights from the failure and dynamic characteristics of two sequential landslides at Baige village along the Jinsha River, China. Landslides, 16(7): 1397-1414.

5. Perski, Z., Hanssen, R., Wojcik, A., Wojciechowski, T. (2009) InSAR analyses of terrain deformation near the Wieliczka Salt Mine, Poland. Eng. Geol., 106(1):58-67.

6. Liu, W., He S., Ouyang C.J. (2016) Dynamic process simulation with a savage-hutter type model for the intrusion of landslide into river. J. Mt. Sci., 13(7):1265-1274. Zeng, X.J. (2017) Monitoring island permafrost deformation over Bei'an-Heihe expressway based on Sentinel-1A data[D]. Harbin: Northeast Forestry University.

7. Xu, H.Y. (2018) Application of InSAR Technology in Subsidence Monitoring along City Roads and Subways-Case of Shenzhen City[D]. Shenzhen: Shenzhen University.

8. Li, Y., Sun, X.P., Li, H.L. (2020) Application of InSAR technology in the analysis and research on the mountain deformation mechanism of a certain expressway in western mountain area [J]. Journal of Geological Hazards and Environment Preservation, 31(03): 88-94.

9. Luo, Y., Tian, Y.F., Zhang, S., Zhang, J.F. (2020) Study on Characteristics of Permafrost Deformation in the Tibetan Plateau Using InSAR Technique[J]. Earthquake, 40(03): 179-188.

10. Yuan, Y., Jia, L.Y., Zhang, X.J., Li, W., Lin, W.J., Wang, C.Q., Hu, D.G. (2020) Land Subsidence Monitoring and Mechanism Analysis in Haikou Based on SBAS-InSAR Technology[J]. Geography and Geo-Information Science, 36(05): 56-64. 